\title{
The Connexion of Duality and Causal Properties for Generalized Free Fields
}

\author{
Wolf-Dieter Garber \\ Institut für Theoretische Physik, Universität Göttingen, \\ Göttingen, Federal Republic of Germany
}

Received September 14, 1974

\begin{abstract}
It is shown that the time-slice axiom and the diamond property are equivalent for the generalized free field. If, in addition, there is a mass gap, duality is equivalent to either causality requirement. It is further shown that the local rings associated with certain space-time regions are factors in the case of causal generalized free fields with mass gap. Necessary and sufficient conditions for causality and duality and some examples for causal and acausal generalized free fields are also given.
\end{abstract}

\section{Introduction}

In their paper on the postulates of quantum field theory [27], Haag and Schroer considered, among other requirements, three restrictions on Wightman fields which they called "primitive causality", "Einstein causality" and "duality". The first two requirements are completeness postulates for the algebra of field operators associated with certain space-time regions:

A field is said to be primitively causal if the von Neumann algebra $R\left(S_{\delta}\right)$ generated by the field operators associated with an arbitrary time-slice $S_{\delta}$ of non-zero thickness $\delta$ already contains all field operators:

$$
R\left(S_{\delta}\right)=R(M), \text { all } \delta>0,
$$

where $M$ denotes Minkowski space. If $(0.1)$ is valid, it is also said that the timeslice axiom holds (see Fig. 1).

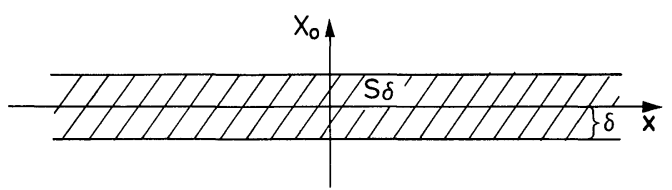

Fig. 1

A field is said to exhibit Einstein causality if the von Neumann algebra of field operators associated with an arbitrary cylinder $Z_{a, \varepsilon}$ contains all field operators associated with the double cone sustended by $Z_{a, \varepsilon}$ :

$$
R\left(Z_{a, \varepsilon}\right)=R\left(Z_{a, \varepsilon}^{\prime \prime}\right) \text { all } a>0, \text { all } \varepsilon>0 .
$$


In this equation, $Z_{a, \varepsilon}^{\prime}$ denotes all points space-like to $Z_{a, \varepsilon}, Z_{a, \varepsilon}^{\prime \prime}=\left(Z_{a, \varepsilon}^{\prime}\right)^{\prime}$, and (0.2) is assumed to hold for all base diameters $a$ and heights $\varepsilon$ (see Fig. 2). In case (0.2) is valid it is also said that the field has hyperbolic propagation character or that the diamond theorem holds.

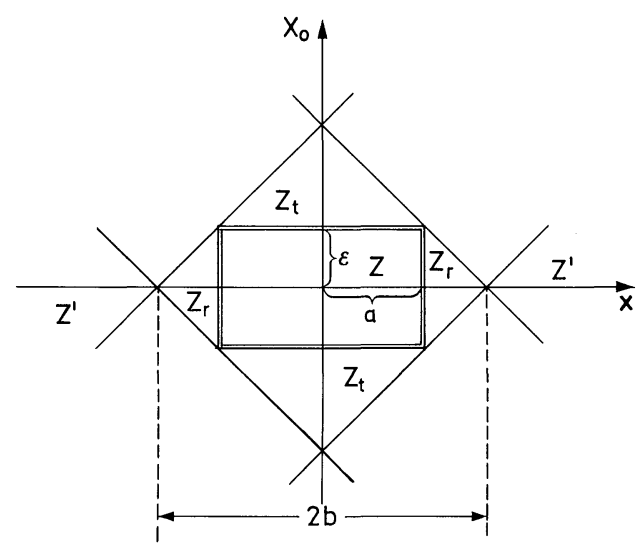

Fig. 2

Duality is a requirement more restrictive than locality; whereas the latter requires field operators to commute whenever they are associated with regions which are situated space-like to each other,

$$
R(X) \subset R^{\prime}\left(X^{\prime}\right) \text { for any region } X,
$$

for duality to hold it is assumed in addition that a field operator commuting with all field operators associated with a given space-time region $X$ is actually localized space-like to $X$ :

$$
R(X)=R^{\prime}\left(X^{\prime}\right) .
$$

Duality does not hold for all regions $X$; a counterexample can be found in [10]. It seems reasonable to restrict the admissible regions for the duality postulate (0.4) to cylinders $Z_{a, \varepsilon}$ and the double cones $Z_{a, \varepsilon}^{\prime \prime}$ sustended by them, and this will be done throughout the paper.

Duality has been proved for the simplest possible case, the free field of mass $m$, in $[10,16,17]$. The proofs all use the diamond property and the time-slice axiom which also hold for the free field.

For the generalized free field, the situation is more complex: [27] contains an example of such a field which is not primitively causal. The same method actually yields a field which does not possess the diamond property and for which, consequently, duality is no longer valid. This poses the problem of investigating the relationship between duality, diamond property, and time-slice axiom in the simplest case where these properties do not necessarily hold. This investigation may conceivably shed some light on the validity of duality in the general case; the renewed interest in this question stems at least partly from the fact that duality 
is a basic assumption in recent work of Doplicher et al. [28] on the superselection structure of quantum fields.

The first section of this paper very briefly lists some well-known properties of generalized free fields and their associated local rings which reduce the study of local rings to the study of the two-point function; the second section is concerned with reducing duality and the causality requirements to equivalent properties of the Kallén-Lehmann-weight of this function thereby investigating the relationship between these properties. In the third section, some simple corollaries and examples are given.

Some time after completing this work, a paper by Landau was published [29] in which duality of generalized free fields is investigated also. It contains a thorough discussion of the relation between duality (which, as also shown by Landau, does not hold for all generalized free fields) and a weak form of duality which is valid for all such fields. This weak duality property allows the construction of a maximal local extension of the system of local algebras such that the extended system will satisfy duality. Though it is mentioned that the methods employed are useful for discussing the time-slice axiom, no result on the relation between diamond property, time-slice axiom and duality is stated or proved. The investigation of the relations between these properties is the main concern of the present paper.

\section{Local Rings for Generalized Free Fields}

In the Wightman framework of quantum field theory [1], generalized free fields are characterized by the fact that the commutator is a complex number. As a consequence, if the one-point function is normalized to zero, the theory is given by the two-point function $[2,7]$ which according to the Wightman axioms and a theorem of Bochner and Martin [9, p. 276], is the Fourier transform of a Lorentz invariant measure of polynomial growth with support in the forward light cone:

$$
(\Omega, A(x) A(y) \Omega)=\int_{\bar{V}_{+}} d \mu(p) \exp (i p(x-y)) .
$$

(For further characterizations of generalized free fields, see [3-6].) The field operators in momentum space, obtained from the Wightman functions via the reconstruction theorem, act on the Fock space over $L^{2}(\mu)$; the corresponding field operators in $x$-space, for real testfunctions $f$ in the Schwartz space $\mathscr{S}$, have stable domains on which they are essentially self-adjoint [8, p. $45 \mathrm{ff}$. $]$. One can therefore define the unitary operator

$$
W(f):=\exp \{i \overline{A(f)}\}
$$

for $f \in \mathscr{S}_{r}$, where the bar denotes operator closure, and the von Neumann algebras

$$
R(X):=\left\{W(f) / f \in \mathscr{D}_{r}(X)\right\}^{\prime \prime}
$$

where $\mathscr{D}_{r}(X)$ is the space of all real, infinitely differentiable functions with compact support in the open space-time region $X$. Duplicating the proof in [10] which uses the Fock space structure of the free field yields the 
Lemma 1.1. The system of von Neumann algebras $\{R(X) / X \subset M$ open $\}$ is a local ring system in the sense of [11].

Since the field operators $A(x)$ have been obtained by Fourier transformation from momentum space, the operators $W(f)$ depend only on $\mathscr{S}_{r} / N$ where $N$ is the space of all $f \in \mathscr{S}_{r}$ whose Fourier transformations have zero $\mu_{+}$-norm, $\mu_{+}$being the symmetric continuation of $\mu$ to $\bar{V}$. On the real space $\mathscr{S}_{r} / N$, a real scalar product can be defined by

$$
(f, g):=\int d \mu_{+}(p) \overline{\hat{f}(p)} g(p)
$$

where the tilde denotes Fourier transformation

$$
\tilde{f}(p):=\int d^{4} x e^{i p x} f(x)
$$

Let $K$ denote the closure of $\mathscr{S}_{r} / N$ in the norm induced by (1.4). One then obtains a representation of the CCR, in Segal's form, over $K$ :

Lemma 1.2. (i) The operators $W(f)$ are defined for all $f \in K$, are unitary and obey the CCR

$$
W(f) W(g)=W(f+g) \exp \left\{\frac{i}{2}(f, \beta g)\right\} .
$$

The operator $\beta: K \rightarrow K$ is defined by

$$
\tilde{\beta f}(p):=i \varepsilon\left(p_{0}\right) \overline{\hat{f}(-p)}
$$

where $\varepsilon$ is the sign function, and obeys

$$
\beta^{2}=-\mathbb{1}, \quad \beta^{*}=-\beta .
$$

(ii) The vacuum functional is given by

$$
(\Omega, W(f) \Omega)=\exp \left\{-\frac{1}{4}(f, f)\right\} .
$$

The proof of this lemma can be found in [12].

As a result of this lemma, von Neumann algebras $R(L)$ can now be defined for an arbitrary closed subspace $L \subset K$ by

$$
R(L):=\{W(f) / f \in L\}^{\prime \prime} .
$$

Consider the lattice of all closed subspaces $K_{\alpha}$ of $K$, and denote the lattice operations by $\vee$ and $\wedge$, where $\wedge K_{\alpha}$ is the intersection of the spaces $K_{\alpha}$ and $\vee K_{\alpha}$ is the closed subspace generated by all finite linear combinations of vectors in the spaces $K_{\alpha}$. The operation $K \mapsto \beta K_{\alpha}^{\perp}\left(K_{\alpha}^{\perp}\right.$ : orthogonal complement of $K_{\alpha}$ in $\left.K\right)$ is a complementation in this lattice. There is a corresponding lattice structure for the von Neumann algebras $R\left(K_{\alpha}\right)$; simply define

$$
\vee R\left(K_{\alpha}\right)=\left(\cup R\left(K_{\alpha}\right)\right)^{\prime \prime} ; \quad \wedge R\left(K_{\alpha}\right):=\cap R\left(K_{\alpha}\right)
$$

and observe that

$$
R \mapsto R^{\prime}
$$

is a complementation. A general theorem of Araki, proved in [13], then states that, due to Lemma 1.2, these two complemented lattices are isomorphic: 
Theorem 1.3. (Araki). The complemented lattices of all closed subspaces of $K$ and of the corresponding von Neumann algebras, defined by (1.10), are isomorphic:

$$
\begin{gathered}
R\left(K_{\alpha}\right) \subset R\left(K_{\beta}\right) \quad \text { iff } \quad K_{\alpha} \subset K_{\beta}, \\
R\left(\vee K_{\alpha}\right)=\vee R\left(K_{\alpha}\right), \\
R\left(\wedge K_{\alpha}\right)=\wedge R\left(K_{\alpha}\right), \\
R^{\prime}\left(K_{\alpha}\right)=R\left(\beta K_{\alpha}^{\perp}\right) .
\end{gathered}
$$

This theorem reduces relations between local rings such as $(0.1),(0.2),(0.3)$, (0.4), to relations between corresponding subspaces of $K$. In a sense,(1.15) is already a sort of duality theorem; however, for $K_{\alpha} \equiv Z_{a, \varepsilon}$, the identification of $\beta K_{\alpha}^{\perp}$ with $Z_{\alpha, \varepsilon}^{\prime}$ remains to be done.

\section{Duality and Causality}

Theorem 1.3 reduces the study of duality and causality to the study of subspaces of $L^{2}\left(\mu_{+}\right)$. By using the Lorentz invariance of the measure $\mu$, the investigation can be simplified still further. In fact, it becomes in essence a single variable problem since a Lorentz invariant measure is uniquely determined by its associated Kallén-Lehmann weight:

Lemma 2.1. Let $\mu$ be a Lorentz invariant measure with support in the closed forward light cone of momentum space, and put $m^{2}:=p^{2}$. Then there is a uniquely determined measure @ of the variable $m$ with support in the semi-axis $[0, \infty)$ such that

$$
d \mu(p)=d \varrho(m) d^{3} \boldsymbol{p} \quad\left(\boldsymbol{p}^{2}+m^{2}\right)^{-1 / 2}
$$

The proof can be found, e.g., in [18, Theorem 2].

For the further discussion, it is convenient to introduce subspaces of $L^{2}(\varrho)$ which correspond to the Fourier transformed subspaces $K(X)$ of $K$ :

Let $\mathscr{D}(\delta)$ be the set of all infinitely differentiable functions with support in the open interval $(-\delta, \delta)$, denote by $\hat{\mathscr{D}}(\delta)$ the Fourier transformed set and by $N(\varrho)$ the set of all $\varrho$-measurable functions with zero $\varrho$-norm. Let $H(\delta)$ be the closure in the $\varrho$-norm of $\tilde{\mathscr{D}}(\delta) / N(\delta)$. Then there is a convenient necessary condition for the time-slice axiom to hold:

Lemma 2.2. Let $\mu$ be the measure of a generalized free field for which the timeslice axiom is valid, and let @ be the Kallén-Lehmann weight associated with it by the previous lemma. Then

$$
H(\delta)=L^{2}(\varrho) \text { for all } \delta>0 .
$$

Proof. Choose $\delta>0$ and pick a function $f$ in the orthogonal complement of $H(\delta)$ in $L^{2}(\varrho)$. In order to show $f=0$, consider the distribution

$$
\hat{f}(x, s):=\int d \mu_{+}(p) \varepsilon\left(p_{0}\right) \exp (i p x) f\left(\left(p^{2}\right)^{1 / 2}\right) \cos \left(s\left(p^{2}\right)^{1 / 2}\right)
$$


with $x \in M, s \in \mathbb{R}$. It is immediate from the definition that $\hat{f} \in \mathscr{S}^{\prime}(x, s)$, and that $\hat{f}$ is a solution of the five-dimensional wave equation

$$
\left(\frac{\partial^{2}}{\partial x_{0}^{2}}-\sum_{i=1}^{3} \frac{\partial^{2}}{\partial x_{i}^{2}}-\frac{\partial^{2}}{\partial s^{2}}\right) \hat{f}(x, s)=0 .
$$

Since the wave operator is hypoelliptic in $x_{0}$, the initial values of $\hat{f}$ on the hyperplane $x_{0}=0$ are well defined distributions in $\mathscr{S}^{\prime}(\boldsymbol{x}, s)$ given by

$$
\begin{aligned}
\hat{f}(0, \boldsymbol{x}, s) & =\int d \mu_{+}(p) \varepsilon\left(p_{0}\right) \exp (-i \boldsymbol{p} \boldsymbol{x}) f\left(\left(p^{2}\right)^{1 / 2}\right) \cos \left(s \cdot\left(p^{2}\right)^{1 / 2}\right), \\
\left.\left(\partial \hat{f} / \partial x_{0}\right)(x, s)\right|_{x_{0}=0} & =\int d \mu_{+}(p)\left|p_{0}\right| \exp (-i \boldsymbol{p} \boldsymbol{x}) f\left(\left(p^{2}\right)^{1 / 2}\right) \cos \left(s \cdot\left(p^{2}\right)^{1 / 2}\right) \\
& =2 i(2 \pi)^{3} \cdot \delta(\boldsymbol{x}) \cdot \int d \varrho(m) f(m) \cos (s \cdot m) .
\end{aligned}
$$

The integral in (2.5) vanishes identically for parity reasons; the integral in (2.6) vanishes if smeared in the variable $s$ with testfunctions in $\tilde{D}(\delta)$ since $f$ is assumed to be orthogonal to $H(\delta)$, i.e. the $x_{0}$-derivative of $f$ vanishes for all $\boldsymbol{x}$ and $|s|<\delta$ in the hyperplane $x_{0}=0$. In fact, all higher $x_{0}$-derivatives of $f$ vanish in this region: the even ones vanish identically, just as $f$ itself does, for parity reasons; the uneven ones are generated by applying powers of the operator $\partial^{2} / \partial x_{0}^{2}$ to $\partial \hat{f} / \partial x_{0}$ which yields zero if (2.4) is combined with (2.6). Hence, $\hat{f}$, together with all its $x_{0^{-}}$ derivatives, vanishes in the region $x_{0}=0,|s|<\delta$. But the support properties of solutions of the wave equation [14, Theorem 7.3] now guarantee that $f$ vanishes in the whole five-dimensional region $\left\{\left(x_{0}, \boldsymbol{x}, s\right) \in \mathbb{R}^{5} /\left|x_{0}\right|+|s|<\delta\right\}$. This means that for $s=0$, the distribution $\hat{f}(x, 0)$ [which is a well defined element of $\mathscr{S}^{\prime}(x)$ because of the $s$-hypoellipticity of the wave operator in (2.4)] vanishes on the time-slice $S_{\delta}:=\left\{x \in M /\left|x_{0}\right|<\delta\right\}$, i.e.:

$$
0=\int d \mu_{+}(p) \varepsilon\left(p_{0}\right) \exp (i p x) f\left(\left(p^{2}\right)^{1 / 2}\right) ; x \in S_{\delta} .
$$

Replace $x$ by $x+a$, where $a=(0, a)$ with $a \in \mathbb{R}^{3}$ is arbitrary, and integrate over $a$ with an arbitrary rotation-invariant test function $k \in \mathscr{S}(\boldsymbol{x})$ to obtain

$$
0=\int d \mu_{+}(p) \varepsilon\left(p_{0}\right) \exp (i p x) f\left(\left(p^{2}\right)^{1 / 2}\right) k(|p|) ; \quad x \in S_{\delta} .
$$

It is easily checked that $f \cdot \hat{k} \in L^{2}\left(\mu_{+}\right)$; smearing (2.8) in $x$ with an arbitrary testfunction $c \in \mathscr{S}(x)$ yields (" $\vee$ " denotes inverse Fourier transformation):

$$
0=\left(\beta c,[f \cdot \hat{k}]^{\vee}\right)
$$

if the definition of $\beta$ in Lemma 1.2 and of the scalar product in $K$ is used. (2.9) means that $[f \cdot \hat{k}]^{\vee} \in \beta K\left(S_{\delta}\right)^{\perp}$; combine Theorem 1.3 with the time-slice axiom (0.1) which holds by assumption to get $f \cdot \tilde{k}=0$ in $L^{2}\left(\mu_{+}\right)$. Now choose $k(x)$ $=\exp \left(-\boldsymbol{x}^{2}\right)$; since its Fourier transform nowhere vanishes, $f=0$ follows.

The condition of this lemma is actually also sufficient; this will follow as a special case of the following lemma which deals with the more general situation of an arbitrary region $X \subset M$ symmetric with respect to the hyperplane $S_{0}:=\left\{x_{0}=0\right\}, X$ being substituted for the time-slices $S_{\delta}$ of the previous lemma. Notice that for such a symmetric region the even and odd parts $f_{+}$and $f_{-}$of a 
function $f \in K(X)$, defined by

$$
f_{ \pm}\left(x_{0}, \boldsymbol{x}\right):=f\left(x_{0}, \boldsymbol{x}\right) \pm f\left(-x_{0}, \boldsymbol{x}\right)
$$

are in $K(X)$, too. With this notation, one obtains

Lemma 2.3. Let $X$ be a region of Minkowski space symmetric with respect to $S_{0}$, and let $f \in \beta K(X)^{\perp}$. Then:

(i) $f_{+}, f_{-} \in \beta K(X)^{\perp}$,

(ii) If, in addition, $H(\delta)=L^{2}(\varrho)$ holds for all $\delta>0$, then, as distributional equations in $\mathscr{D}^{\prime}\left(X \cap S_{0}\right)$,

$$
\begin{gathered}
\int d^{3} \boldsymbol{p} \exp (-i \boldsymbol{p} \cdot \boldsymbol{x}) f_{+}\left(\boldsymbol{p}^{2}+m^{2}, \boldsymbol{p}\right)=0, \\
\int d^{3} \boldsymbol{p} \exp (-i \boldsymbol{p} \cdot \boldsymbol{x})\left(\boldsymbol{p}^{2}+m^{2}\right)^{-1 / 2} f_{-}\left(\left(\boldsymbol{p}^{2}+m^{2}\right)^{1 / 2}, \boldsymbol{p}\right)=0
\end{gathered}
$$

for $\varrho$-almost all $m$.

Proof. (i) $f \in \beta K(X)^{\perp}$ is equivalent to $(f, \beta g)=0$ for all $g \in K(X)$. This is true, in particular, for all even and odd parts $g_{+}, g_{-}$of functions $g \in K(X)$ :

$$
\left(f, \beta g_{+}\right)=0=\left(f, \beta g_{-}\right)
$$

which are in $K(X)$ since $X$ is symmetric. Subtracting from this the equations

$$
\left(f_{+}, \beta g_{+}\right)=0=\left(f_{-}, \beta g_{-}\right)
$$

which hold for parity reasons by definition of $\beta$, yields

$$
\left(f_{-}, \beta g_{+}\right)=0=\left(f_{+}, \beta g_{-}\right) \text {. }
$$

Reading (2.15) from right to left and adding it to (2.14) gives

$$
\left(f_{+}, \beta g\right)=0=\left(f_{-}, \beta g\right),
$$

i.e. $f_{+}, f_{-} \in \beta K(X)^{\perp}$.

(ii) First consider $f_{+}$and define, analogously to (2.3),

$$
\hat{f}_{+}(x, s):=\int d \mu_{+}(p) \varepsilon\left(p_{0}\right) \exp (i p x) f_{+}(p) \cos \left(s\left(p^{2}\right)^{1 / 2}\right) ;
$$

$\hat{f}_{+}$is a solution of $(2.4)$ in $\mathscr{S}^{\prime}(x, s)$; its $x_{0}$-derivative on $S_{0}$ is given by

$$
\begin{aligned}
\left.\left(\partial \hat{f}_{+} / \partial x_{0}\right)(x, s)\right|_{x_{0}=0} & =i \int d \mu_{+}(p)\left|p_{0}\right| \exp (-i \boldsymbol{p} \boldsymbol{x}) f_{+}(p) \cos \left(s \cdot\left(p^{2}\right)^{1 / 2}\right) \\
& =2 i \int d \varrho(m) d^{3} \boldsymbol{p} \exp (-i \boldsymbol{p} \boldsymbol{x}) f_{+}\left(\boldsymbol{p}^{2}+m^{2}, \boldsymbol{p}\right) \cos (s \cdot m) .
\end{aligned}
$$

Notice that $\hat{f}_{+}$and all derivatives of $\hat{f}_{+}$with respect to $s$ and $x_{k}, k=1,2,3$ vanish for $s=0$ and $x \in X$ : the odd $s$-derivatives vanish for $s=0$ anyway because of the factor $\sin \left(s\left(p^{2}\right)^{1 / 2}\right)$ which appears in the integrand; the even $s$-derivatives and all $x_{k}$-derivatives vanish since $f_{+} \in \beta K(X)^{\perp}$.

Assume that $X \cap S_{0}$ is not empty (otherwise the assertions are trivial), and choose an arbitrary point $(0, x) \in X \cap S_{0}$. Since $X$ is open, there exists a positive number $\delta$ depending on $\boldsymbol{x}$ and open balls $U_{2 \delta}$ in $\mathbb{R}^{3}$ (with center $\boldsymbol{x}$ and radius $2 \delta$ ) and $U_{2 \delta}^{\prime}$ in $\mathbb{R}^{1}$ (with center $x_{0}=0$ and radius $2 \delta$ ) such that the Cartesian product $U=U_{2 \delta}^{\prime} \times U_{2 \delta}$ is in $X$. Again the support properties of solutions of the wave 
equation (2.4) [14, Theorem 7.2] guarantee that the vanishing of $\hat{f}_{+}$, together with all $s$-derivatives, for $s=0$ and $x \in U$ implies $\hat{f}_{+}=0$ for $x \in U_{2 \delta}$ and $\left|x_{0}\right|+|s|<2 \delta$; simply consider any time-like curve in $U$ and notice that $\hat{f}_{+}$must vanish on the double cone sustended by that curve. $\hat{f}_{+}=0$ implies $\partial \hat{f}_{+} / \partial x_{0}=0$ in the same region; in particular, for $x_{0}=0, \partial \hat{f}_{+} / \partial x_{0}=0$ for $|s|<2 \delta$. Integrating (2.17) with an arbitrary test function $g \in \mathscr{D}\left(U_{2}\right)$ yields

$$
0=\int d \varrho(m) \cos (s \cdot m) F_{+}(m, g) \text { for }|s|<2 \delta
$$

where

and

$$
F_{+}(m, g):=\int d^{3} \boldsymbol{x} g(\boldsymbol{x}) F_{+}(m, \boldsymbol{x})
$$

$$
F_{+}(m, \boldsymbol{x}):=\int d^{3} \boldsymbol{p} \exp (-i \boldsymbol{p} \boldsymbol{x}) f_{+}\left(\boldsymbol{p}^{2}+m^{2}, \boldsymbol{p}\right) .
$$

Smearing (2.18) in $s$ with a testfunction obtained as the convolution product of $\cos (t \cdot m)$ in $t$ and an arbitrary function $h \in \mathscr{D}(\delta)$ results in

$$
0=\int d \varrho(m) \cos (t \cdot m) \hat{h}(m) F_{+}(m, g) \text { for }|t|<\delta .
$$

Since $\hat{h} \cdot F_{+} \in L^{2}(\varrho)$, the assumption $H(\delta)=L^{2}(\varrho)$ implies $\hat{h} \cdot F_{+}=0 \varrho-$ a.e., for arbitrary $h \in \mathscr{D}(\delta)$. Notice that $\tilde{\mathscr{D}}(\delta)$ contains, along with a function $h$, also the translated function $h_{a}$, defined by $h_{a}(m):=h(m+a)$ where $a \in \mathbb{R}^{1}$ is arbitrary. As $\tilde{\mathscr{D}}(\delta) \neq\{0\}$, to every point $m_{0}$ one can find a function $\hat{h}^{\left(m_{0}\right)} \in \tilde{\mathscr{D}}(\delta)$ which is not zero in $m_{0}$. Hence $F_{+}(m, g)=0 \varrho$-a.e. which implies in particular $F_{+}\left(m, g_{v}\right)=0 \varrho$-a.e. for any countable base $\left\{g_{\nu}\right\}$ of $\mathscr{D}\left(U_{\delta}\right)$. Since $F_{+}(m, g)$ is continuous in $g$ [as a linear functional on $\left.\mathscr{D}^{\prime}\left(U_{\delta}\right)\right]$, it follows that $F_{+}(m, x)=0 \varrho$-a.e. as a distribution in $\mathscr{D}^{\prime}\left(U_{\delta}\right)$. As the neighbourhood $U_{\delta}$ was chosen around an arbitrary point $\boldsymbol{x}$ such that $(0, x) \in S_{0} \cap X,(2.11)$ follows.

In the case of $f_{-}$, the proof proceeds similarly, with $f_{+}$replaced by $f_{-}$ throughout. The only other change in the argument occurs in the lines just before (2.18) where $f_{-}$is now evaluated on $S_{0}$ instead of its $x_{0}$-derivative (which would be identically zero for parity reasons). This accounts for the extra factor of $p_{0}$ $=\left(p^{2}+m^{2}\right)^{1 / 2}$ in (2.12) which does not cancel.

The lemma just proven is, in a way, the essence of all arguments which follow; by allowing statements for fixed $m$ in the support of $\varrho$, it achieves the reduction to the free field situation (for which an independent proof of duality will be provided, however). As an immediate corollary, the second part of the lemma shows that the necessary condition (2.2) of Lemma 2.2 is also sufficient for the time-slice axiom to hold; hence

Theorem 2.4. Let $\mu$ be the measure of a generalized free field and $\varrho$ be its associated Kallén-Lehmann weight. Then the time-slice axiom holds if and only if

$$
H(\delta)=L^{2}(\varrho) \text { for all } \delta>0 .
$$

Now the relation between the time-slice axiom and the diamond property can be investigated. Even for a general local ring system, the latter implies the former as is easily seen by taking, for an arbitrary but fixed time-slice $S_{\delta}$, a sequence of cylinders $Z_{n}$ such that $\cup Z_{n}=S_{\delta}$. For generalized free fields, the converse is also true: 
Theorem 2.5. A generalized free field has the diamond property if and only if it is primitively causal.

Proof. Assume that the time-slice axiom holds, i.e. that $H(\delta)=L^{2}(\varrho)$ for all $\delta>0$ by Lemma 2.2. Choose an arbitrary cylinder

$$
Z_{a, \varepsilon}:=\left\{x \in M /\left|x_{0}\right|<\varepsilon,|x|<a\right\}
$$

and a function $f \in \beta K\left(Z_{a, \varepsilon}\right)^{\perp}$. Now by a general result of Borchers [15], $R\left(Z_{a, \varepsilon}\right)$ $=R\left(Z_{a, \varepsilon} \cup Z_{r}\right)$, see Fig. 2 for the notation, which implies by Theorem 1.3 that $f \in \beta K\left(Z_{a, \varepsilon} \cup Z_{r}\right)^{\perp}$. Lemma 2.3, for $X=Z_{a, \varepsilon} \cup Z_{r}$ now yields

$$
\begin{gathered}
\int d^{3} \boldsymbol{p} \exp (-i p \boldsymbol{x}) f_{+}\left(\boldsymbol{p}^{2}+m^{2}, \boldsymbol{p}\right)=0 \\
\int d^{3} \boldsymbol{p}\left(\boldsymbol{p}^{2}+m^{2}\right)^{-1 / 2} \exp (-i \boldsymbol{p} \boldsymbol{x}) f_{-}\left(\left(p^{2}+m^{2}\right)^{1 / 2}, \boldsymbol{p}\right)=0
\end{gathered}
$$

for $\varrho$-almost all $m$ and $|x|<a+\varepsilon$. Integrating the first equation over $m$ with weight $d \varrho(m) \cdot \cos (s \cdot m)$ shows that the initial values on $x_{0}=0$ of the distribution $\hat{f}_{+}$ obtained from $f_{+}$by the defining Eq. (2.16) vanish for $|x|<a+\varepsilon$ and all $s$. As explained in Lemma 2.3, this implies that $\hat{f}_{+}$vanishes in $\left|x_{0}\right|+|x|<a+\varepsilon$, i.e. in $Z_{a, \varepsilon}^{\prime \prime}$ which means that $f_{+} \in \beta K\left(Z_{a, \varepsilon}^{\prime \prime}\right)^{\perp}$. Analogously it is shown that $f_{-} \in \beta K\left(Z_{a, \varepsilon}^{\prime \prime}\right)^{\perp}$ implying $f \in \beta K\left(Z_{a, \varepsilon}^{\prime \prime}\right)^{\perp}$. By Theorems 1.3 and Lemma 1.1, the inclusion

$$
\beta K\left(Z_{a, \varepsilon}\right)^{\perp} \subset \beta K\left(Z_{a, \varepsilon}^{\prime \prime}\right)^{\perp}
$$

is equivalent to the diamond property $(0.4)$ for $Z_{a, \varepsilon}$.

Henceforth, generalized free fields which are primitively causal or have the (equivalent) property of Einstein causality will simply be called "causal".

To investigate the relationship between duality and causality, notice first that, even for a general local ring system, duality for a cylinder $Z, R(Z)=R^{\prime}\left(Z^{\prime}\right)$, implies the diamond property: Since $Z^{\prime \prime} \supset Z, R\left(Z^{\prime \prime}\right) \supset R(Z)=R^{\prime}\left(Z^{\prime}\right)$, whereas locality implies the converse inclusion $R\left(Z^{\prime \prime}\right) \subset R^{\prime}\left(Z^{\prime}\right)$. Comparing $R\left(Z^{\prime \prime}\right)=R^{\prime}\left(Z^{\prime}\right)$ to the duality equation for $Z$ yields $R\left(Z^{\prime \prime}\right)=R(Z)$, the diamond property for $Z$.

At least for generalized free fields with a mass gap, the converse is also true. It is necessary, however, to recall first some properties of local Sobolev spaces of fractional index which will be used in the proof:

For the definition of the global Sobolev spaces, consider the operator

$$
M^{t}:=\left(-\Delta+m^{2}\right)^{t / 2}
$$

(where $\Delta$ is the Laplacian, $m>0$ is a fixed number, and $t \in \mathbb{R}$ is arbitrary) acting on the space $L_{r}^{2}$ of all real, Lebesgue square integrable functions in $\mathbb{R}^{3}$ with scalar product ( , ). Let its domain of definition be $D\left(M^{t}\right)$ and denote by $L_{+}$the completion of $D\left(M^{1 / 2}\right) \cap L_{r}^{2}$ with respect to the scalar product $(,)_{+}$given by

$$
(f, g)_{+}:=\left(M^{1 / 2} f, M^{1 / 2} g\right)
$$

and by $L_{-}$the completion of $L_{r}^{2}$ with respect to the additional scalar product $(,)_{-}$defined by

$$
(f, g)_{-}:=\left(M^{-1 / 2} f, M^{-1 / 2} g\right) .
$$

$L_{+}$and $L_{-}$are global Sobolev spaces of fractional index $+(1 / 2)$ resp. $-(1 / 2)$. To obtain the corresponding local spaces, consider a measurable set $B \subset \mathbb{R}^{3}$ with 
non-empty interior int $B$, and define

$$
\begin{aligned}
& L_{+}(B):=\left\{f \in L_{+} / \operatorname{supp} f(\operatorname{int} B\},\right. \\
& L_{-}(B):=\left\{g \in L_{-} /\left(M^{-1 / 2} g, M^{1 / 2} f\right)=0 \text { for all } f \in L_{+}\left(B^{c}\right)\right.
\end{aligned}
$$

where $B^{c}$ denotes the complement of $B$ in $\mathbb{R}^{3}$. With the use of the isometric mappings

$$
M^{1 / 2}: L_{+} \rightarrow L^{2} ; \quad M^{-1 / 2}: L_{-} \rightarrow L^{2}
$$

corresponding subspaces of $L_{r}^{2}$ can be defined as

$$
N_{+}(B):=M^{1 / 2} L_{+}(B) ; \quad N_{-}(B):=M^{-1 / 2} L_{-}(B) ;
$$

for a general set $B \subset \mathbb{R}^{3}, N_{+}(B)$ and $N_{-}(B)$ denote the intersection of all spaces $N_{+}\left(B_{1}\right)$ and $N_{-}\left(B_{1}\right)$ where $B_{1}$ runs over all regions with non-empty interior containing $B$.

For these spaces, various relations hold which are proved in [10] and [19], and of which only the ones used later are listed below for convenience.

Lemma 2.6. Let $B, B_{1}, B_{2}$ be regions in $\mathbb{R}^{3}$ with non-empty interior, and assume their boundaries $\partial B, \partial B_{1}, \partial B_{2}$ to be piecewise infinitely often differentiable two dimensional surfaces. Then

(i) $N_{+}(B)^{\perp}=N_{-}\left(B^{c}\right) ; \quad N_{-}(B)^{\perp}=N_{+}\left(B^{c}\right)$,

(ii) $N_{+}(\bar{B})=N_{+}(B) ; \quad N_{-}(\bar{B})=N_{-}(B)$,

(iii) $N_{+}(\partial B)=\{0\} ; \quad N_{-}(\partial B)=\{0\}$ ，

(iv) $N_{+}\left(B_{1}\right) \cap N_{+}\left(B_{2}\right)=N_{+}\left(B_{1} \cap B_{2}\right) ; \quad N_{-}\left(B_{1}\right) \cap N_{-}\left(B_{2}\right)=N_{-}\left(B_{1} \cap B_{2}\right)$.

Relations such as (2.27) to (2.30) do not hold for general sets, see [19]; nor do they hold for Sobolev spaces with general fractional index, see [20]. However, for generalized free fields only the indices $(+1 / 2)$ and $(-1 / 2)$ accur in which cases Lemma 2.6 holds and permits the proof of duality:

Theorem 2.7. Let $\mu$ be the measure of a causal generalized free field, and assume in addition that $\mu$ has a mass gap, i.e. that

$$
E_{0}:=\inf \left\{p_{0} \in \mathbb{R} /\left(p_{0}, \boldsymbol{p}\right) \in \operatorname{supp} \mu, p_{0} \neq 0\right\}>0
$$

or equivalenty (see Lemma 2.1)

$$
E_{1}:=\inf \{m \in \mathbb{R} / m \in \operatorname{supp} \varrho, m \neq 0\}>0 .
$$

Then the duality equation (0.4) holds for every cylinder $Z$.

Proof. 1. Choose an arbitrary cylinder $Z=Z_{a, \varepsilon}$; by locality and Borchers' result [15], it is enough to prove $R\left(Z^{\prime}\right) \supset R^{\prime}\left(Z \cup Z_{r}\right)$ which reduces to $K\left(Z^{\prime}\right)$ $\supset \beta K\left(Z \cup Z_{r}\right)^{\perp}$ by Theorem 1.3. Choose $f \in \beta K\left(Z \cup Z_{r}\right)^{\perp}$; by the symmetry of $Z \cup Z_{r}$, Lemma 2.3 yields $f_{+}, f_{-} \in \beta K\left(Z \cup Z_{r}\right)^{\perp}$.

Consider first $f_{+}$and notice that the equation

$$
\left(f_{+}, \beta g\right)=0
$$


had it been proved for all $g \in J:=\beta K\left(Z^{\prime}\right)^{\perp}$, would imply $f \in \beta J^{\perp}=K\left(Z^{\prime}\right)$, as required. But $\left(f_{+}, \beta g_{+}\right)=0$ for all $g_{+} \in K$ for parity reasons, hence it is sufficient to prove

$$
\left(f_{+}, \beta g_{-}\right)=0
$$

for arbitrary $g_{-} \in J$.

2. Lemma 2.3 implies for $f_{+} \in \beta K\left(Z \cup Z_{r}\right)^{\perp}$ and $g_{-} \in J=\beta K\left(Z^{\prime}\right)^{\perp}$ :

$$
\begin{gathered}
F_{+}(m):=\int d^{3} \boldsymbol{p} \exp (-i \boldsymbol{p} \boldsymbol{x}) f_{+}\left(\boldsymbol{p}^{2}+m^{2}, \boldsymbol{p}\right)=0,|\boldsymbol{x}|<b, \\
G_{-}(m):=\int d^{3} \boldsymbol{p} \exp (-i \boldsymbol{p} \boldsymbol{x}) g_{-}\left(\left(p^{2}+m^{2}\right)^{1 / 2}, \boldsymbol{p}\right) \cdot\left(\boldsymbol{p}^{2}+m^{2}\right)^{-1 / 2}=0|\boldsymbol{x}|>b
\end{gathered}
$$

for $\varrho$-almost every $m$, (see Fig. 2 for the meaning of $b=a+\varepsilon$ ). Define $K_{b}:=\left\{\boldsymbol{x} \in \mathbb{R}^{3} /|\boldsymbol{x}|<b\right\}$, for abbreviation.

Now choose an arbitrary, but fixed $m>0$; it is immediate from the definition of $F_{+}$and $G_{-}$that $F_{+} \in L_{-}, G_{-} \in L_{+}$. (2.34) and (2.35) show furthermore, that $F_{+} \in L_{-}\left(\left(K_{b}\right)^{c}\right), G_{-} \in L_{+}\left(K_{b}\right)$ which gives, by Lemma 2.6,

$$
\begin{gathered}
M^{-1 / 2} F_{+} \in N_{-}\left(\left(K_{b}\right)^{c}\right)=N_{+}\left(K_{b}\right)^{\perp}, \\
M^{1 / 2} G_{-} \in N_{+}\left(\bar{K}_{b}\right)=N_{+}\left(K_{b}\right),
\end{gathered}
$$

hence

$$
\begin{aligned}
0 & =\int d^{3} \boldsymbol{x}\left(M^{1 / 2} G_{-}\right)\left(M^{-1 / 2} F_{+}\right)=\int d^{3} p G_{-} \cdot F_{+} \\
& =\int d^{3} \boldsymbol{p} f_{+}\left(\boldsymbol{p}^{2}+m^{2}, \boldsymbol{p}\right) g_{-}\left(\left(\boldsymbol{p}^{2}+m^{2}\right)^{1 / 2}, \boldsymbol{p}\right)\left(\boldsymbol{p}^{2}+m^{2}\right)^{-1 / 2}
\end{aligned}
$$

if Parseval's equation and (2.34), (2.35) are used. Integrating the last equation over $m$ with weight $\varrho$, which, because of the mass gap, is an integration over $m>0$, finally yields

$$
0=\int d \mu(p) f_{+}(p) g_{-}(p)=2^{-1} \int d \mu(p) \varepsilon\left(p_{0}\right) f_{+}(p) g_{-}(p)
$$

which is (2.33).

The same technique of proof actually shows that the rings $R(Z), R\left(Z^{\prime \prime}\right)$ for the causal generalized free fields are factors:

Theorem 2.8. Let $X \subset M$ be a region symmetric with respect to the hyperplane $S_{0}:=\left\{x_{0}=0\right\}$, and assume $X \cap S_{0}=K_{a}, X^{\prime} \cap S_{0}=\operatorname{int}\left(K_{a}\right)$, where

$$
K_{a}:=\left\{\boldsymbol{x} \in \mathbb{R}^{3} /|\boldsymbol{x}|<a\right\} .
$$

Then the local ring $R(X)$ for a generalized free field with a mass gap is a factor.

Proof. Choose $f \in K(X) \cap \beta K(X)^{\perp}$; by Theorem 1.3 it suffices to show $f=0$. By Lemma $2.3, f_{+}, f_{-} \in \beta K(X)^{\perp}$ which implies as in the previous proof that, for every fixed $m>0$

$$
\begin{gathered}
F_{+}(\boldsymbol{x}):=\int d^{3} \boldsymbol{p} \exp (-i \boldsymbol{p} \boldsymbol{x}) f_{+}\left(\boldsymbol{p}^{2}+m^{2}, \boldsymbol{p}\right) \in L_{-}\left(\left(K_{a}\right)^{c}\right) \\
F_{-}(\boldsymbol{x}):=\int d^{3} \boldsymbol{p} \exp (-i \boldsymbol{p} \boldsymbol{x}) f_{-}\left(\left(\boldsymbol{p}^{2}+m^{2}\right)^{1 / 2}, \boldsymbol{p}\right)\left(\boldsymbol{p}^{2}+m^{2}\right)^{-1 / 2} \in L_{+}\left(\left(K_{a}\right)^{c}\right) .
\end{gathered}
$$

Hence

$$
M^{1 / 2} F_{-} \in N_{+}\left(\left(K_{a}\right)^{c}\right), \quad M^{-1 / 2} F_{+} \in N_{-}\left(\left(K_{a}\right)^{c}\right)
$$


$f_{+}, f_{-} \in K(X)$ implies $f_{+}, f_{-} \in \beta K\left(X^{\prime}\right)^{\perp}$, by locality, which shows, by the same reasoning as above that

$$
M^{1 / 2} F_{-} \in N_{+}\left(\bar{K}_{a}\right), \quad M^{-1 / 2} F_{+} \in N_{-}\left(\bar{K}_{a}\right)
$$

(2.36) and (2.37) imply, by Lemma 2.6, that

$$
M^{1 / 2} F_{-} \in N_{+}\left(\partial K_{a}\right)=\{0\}, \quad M^{-1 / 2} F_{+} \in N_{-}\left(\partial K_{a}\right)=\{0\}
$$

which gives $f_{+}=0=f_{-}$by Fourier transformation.

The proof of duality and the factor property for generalized free fields as given above uses in detail the structure of Sobolev spaces which arise from the Lorentz invariance of the measure $\mu$; a proof based upon only the finite Lorentz covariance of $\mu$ guaranteed by the work in [23] would require major modifications.

\section{Conditions and Examples for Causal Fields}

The results of Section 2 show that duality, diamond property and time-slice axiom are all equivalent for a generalized free field with mass gap. Theorem 2.4 even gives a characterization of causal fields in terms of the defining measures $\mu$ which is, however, rather an implicit one. In this section, conditions equivalent to (2.2) will be listed which are somewhat easier to handle.

Equations such as (2.2) play a certain role in probability and prediction theory and have been investigated before [21, 22, 24]. Chapter 8 of [21] contains a rather neat condition, which, for a restricted class of measures $\varrho$, is equivalent to (2.2):

Lemma 3.1. Let $\varrho$ be a measure on $\mathbb{R}_{+}$with $\int_{0}^{\infty} d \varrho(m)<\infty$, and assume there exists a number $m_{0} \geqq 0$ such that for $m \geqq m_{0}$

(i) @ decreases monotonically,

(ii) $\varrho$ is absolutely continuous with respect to Lebesgue measure $d m$.

Then the measure $\mu$ constructed from @ via Eq. (2.1), is the measure of a causal generalized free field if and only if

$$
\int_{m_{0}}^{\infty} d m \cdot m^{-2} \log (d \varrho / d m)=-\infty .
$$

For arbitrary measures, there exist conditions equivalent to (2.2) which, however, explicitly depend on $\delta$ and are thus less convenient, see [22].

Lemma 3.1 leads directly to examples of causal and acausal fields; thus, a measure $\varrho$ which can be represented as

$$
d \varrho(m)=d m \exp \left(-\left(m^{\alpha}\right)\right)
$$

for large $m$, leads to a causal field for $\alpha \geqq 1$ and to an acausal one for $\alpha<1$.

A special class of generalized free fields are those which can be defined, together with all $x_{0}$-derivatives, on a time-slice of zero thickness. This class contains precisely those fields for which the moments of the corresponding measure $\varrho$ are finite. A sufficient condition for these fields to be causal is given in the following

Lemma 3.2. Assume that all moments

$$
c_{n}:=\int_{0}^{\infty} d \varrho(m) m^{n} \quad n \in \mathbb{N}
$$


of the measure @ are finite and that the moment problem defined by (3.2) has a unique solution; then the generalized free field constructed from @ is causal.

Proof. To prove $H(\delta)=L^{2}(\varrho)$ for all $\delta>0$, fix $\delta$ and choose $f \in H(\delta)^{\perp}$, i.e.

$$
\int_{0}^{\infty} d \varrho(m) e^{i s m} f(m)=0 \text { for }|s|<\delta
$$

differentiate $n$-times at $s=0$ to obtain

$$
\int_{0}^{\infty} d \varrho(m) \cdot m^{n} \cdot f(m)=0, \quad n \in \mathbb{N}
$$

and use the fact, which is proved in [25, p. 45] that the polynomials are dense in $L^{2}(\varrho)$ whenever $\varrho$ defines a determined moment problem, to conclude $f=0$, as required.

There are several necessary, sufficient and equivalent conditions for a moment problem to have a unique solution, see $[25,26]$. A rather simple sufficient criterion is

$$
\sum_{n}\left(c_{n}\right)^{-1 / 2 n}=\infty
$$

which means roughly that the moments should not increase too fast [26, p. 19].

Additional conditions beyond the existence of the moments (3.2) are indeed needed to guarantee causality: Chapter 11 of [21] contains an example of a measure with finite moments such that $H(\delta) \neq L^{2}(\varrho)$ for all $\delta>0$.

Acknowledgments. The author wishes to thank Professor Dr. H. J. Borchers for proposing the problem to him and for numerous helpful discussions, and Professor Dr. H. Reeh for constructive criticism and hints.

\section{References}

1. Wightman,A.S.: Quantum field theory in terms of vacuum expectation values Phys. Rev. 101, 860 (1956)

2. Greenberg, O.W.: Generalized free fields and models of local field theory. Ann. Phys. 16, 158 (1961)

3. Licht, A.L., Toll, J.S.: Two-point function and generalized free fields. Nuovo Cimento 21, 346 (1961)

4. Robinson, D. W.: Support of a field in momentum space. Helv. Phys. Acta 35, 403 (1962)

5. Greenberg, O. W.: Heisenberg fields which vanish on domains of momentum space. J. Math. Phys. 3, 859 (1962)

6. Robinson, D.W.: A theorem concerning the positive metric. Commun. math. Phys. 1, 89 (1965)

7. Borchers, H.J.: Einführung in die Quantenfeldtheorie I, II. Lecture Notes 1966/67 University of Göttingen

8. Jost, R.: The general theory of quantized fields. Providence: American Math. Soc. 1965

9. Schwartz, L.: Théorie des Distributions. Paris: Hermann 1966

10. Araki,H.: Von Neumann algebras of local observables for free scalar fields. J. Math. Phys. 5, 1 (1964)

11. Haag, R., Kastler, D.: An algebraic approach to quantum field theory. J. Math. Phys. 5, 848 (1964)

12. Streit, L.: Generalized free fields as cyclic representations of the canonical commutation relations. Acta Phys. Austriaca 32, 107 (1970)

13. Araki,H.: A lattice of von Neumann algebras associated with the quantum theory of a free Bose field. J. Math. Phys. 4, 1343 (1963) 
14. Wightman,A.S.: Analytic functions of several complex variables. In: Rélations de dispersion et particules élémentaires ed.: de Witt/Omnès, Paris: Hermann 1960

15. Borchers, H. J.: On the completeness of Lorentz invariant fields in a timelike tube. Nuovo Cimento 19, 787 (1961)

16. dell'Antonio, G.F.: Structure of the algebras of some free systems. Commun. math. Phys. 9, 81 (1968)

17. Osterwalder, K.: Duality for free Bose fields. Commun. math. Phys. 29, 1 (1973)

18. Güttinger, W., Rieckers, A.: Spectral representations of Lorentz invariant distributions and scale transformation. Commun. math. Phys. 7, 190 (1968)

19. Strichartz, R.S.: Mulipliers on fractional Sobolev spaces. J. Math. Mech. 16, 1031 (1967)

20. Donoghue, W.F.: Distributions and Fourier transforms. New York and London: Academic Press (1969)

21. Levinson, N., McKean,H.P.: Weighted trigonometrical approximation on $R^{1}$ with application to the germ field of a stationary Gaussian noise. Acta Math. 112, 99 (1964)

22. Akutowicz, J.: Sur l'approximation par certaines fonctions entières. Ann. Sci. Ec. Norm. Sup. (3) 77, $281(1960)$

23. Bros, J., Epstein,H., Glaser, V.: On the connection between analyticity and Lorentz covariance of Wightman functions. Commun. math. Phys. 6, 77 (1967)

24. Achieser, N.I.: Vorlesungen über Approximationstheorie. Berlin: Akademie-Verlag (1967)

25. Achieser, N.I.: The classical moment problem and some related questions in analysis. Edinburgh and London: Oliver and Boyd (1965)

26. Shohat, J.A., Tamarkin, J. D.: The problem of moments. New York: Am. Math. Soc. (1943)

27. Haag, R., Schroer, B.: The postulates of quantum field theory. J. Math. Phys. 3, 248 (1962)

28. Doplicher, S., Haag, R., Roberts, J. : Field observables and gauge transformations I, II. Commun. math. Phys. 13, 1 (1969) Commun. math. Phys. 15, 173 (1969)

29. Landau, L. J.: On local functions of fields. Commun. math. Phys. 39, 49 (1974)

Communicated by H. Araki

\author{
Wolf-Dieter Garber \\ Institut für Theoretische Physik \\ Universität Göttingen \\ D-3400 Göttingen \\ Bunsenstraße 9 \\ Federal Republic of Germany
}

